\title{
Chang-Kang-Fang ameliorates irritable bowel syndrome with diarrhea in rat by regulating gut microbiota and improving intestinal barrier
}

\author{
Min Lu ${ }^{\mathrm{a}, \mathrm{b}, \dagger}$, Xinyu Fan ${ }^{\mathrm{c}, \mathrm{d}, \dagger}$, Yuanyuan Zheng ${ }^{\mathrm{e}}$, Weiqian Kong ${ }^{\mathrm{e}}$, Ruyi Ji ${ }^{\mathrm{e}}$, Hui Xie ${ }^{\mathrm{e}, *}$ \\ a Affiliated Hospital of Integrated Traditional Chinese and Western Medicine, Nanjing University of Chinese \\ Medicine, Nanjing 210023 China \\ b Jiangsu Province Academy of Traditional Chinese Medicine, Nanjing 210023 China \\ c Nanjing Lishui District Hospital of Traditional Chinese Medicine, Nanjing 210023 China \\ d Clinical College of Jiangsu Health Vocational College, Nanjing 210023 China \\ e The Third Clinical Medical College, Nanjing University of Chinese Medicine, Nanjing 210023 China \\ *Corresponding author, e-mail: xiehuitcm@163.com \\ $\dagger$ These authors contributed equally to this work.
}

\begin{abstract}
Chang-Kang-Fang formula (CKF), a multi-herb traditional Chinese medicinal formula, has been clinically used for treatment of irritable bowel syndrome with diarrhea (IBS-D). Though we have reported the compounds of CKF and their therapeutic effect on IBS-D rats, the exact underlying mechanism is still not clear. The aim of this study was to clearly define the effect of CKF on IBS-D by regulating gut microbiota and promoting intestinal barrier. IBS-D rats were established by psychosocial stress (restraint) combined with the peripheral stimulation (senna leaf gavage) stress. The changes of body weight and the number of fecal pellets were investigated during the experiment. The effect on intestinal sensitivity was assessed based on the abdominal withdrawal reflex (AWR) scores and the intestinal permeability, the expression of zona occludens 1 (ZO-1), measured by immunohistochemistry. The composition of gut microbiota was detected through $16 \mathrm{~S}$ rRNA. The administration of CKF significantly had therapeutic effects on IBS-D rats based on the decreased AWR scores and the increased number of pellets. However, the changes of body weight were not observed. In addition, CKF could up-regulate the expression of ZO-1 in the colon and rebalance the gut microbiota of IBS-D by increasing the abundance of Lactobacillus, Allobaculum, Roseburia, and Lachnospiraceae_NK4A136. CKF potentially alleviated IBS-D through regulating gut microbiota and improving the intestinal barrier function.
\end{abstract}

KEYWORDS: gut microbiota, diarrhea predominant irritable bowel syndrome, TCM

\section{INTRODUCTION}

Irritable bowel syndrome (IBS) is a disease of functional disorders of the gastrointestinal tract characterized by abdominal pain and changes of defecation, leading to a decline in quality of life. According to the predominant feature of the bowel symptoms, IBS can be divided into four subtypes: IBS with constipation (IBS-C), IBS with diarrhea (IBS-D), mixed-type IBS (IBS-M), and unclassified IBS (IBS-U). Prevalence of IBS varies between $1.1 \%$ and $45 \%$, with a pooled global prevalence of $11 \%[1,2]$. Among the four subtypes, IBS-C and IBS-D each accounts for one-third of the affected population [2]. Though the mechanism of IBS is still unknown, dysfunction of intestinal motility, hypersensitivity of the colon, weakness of tight junctions, and imbalance of gut microbiota may play vital roles in IBS-D [3, 4]. Among all the factors, the crosstalk between gut microbiota and host in intestinal epithelium has attracted attention. With the development of microbiology, dysregulated microbiota in IBS-D has been reported, and researchers have done plenty of work to reveal the mechanism of IBS-D based on this view. Various factors, such as diet, environment, and antibiotics treatment, may affect the structure of gut microbiota, leading to dysfunction of the flora. The intestinal epithelium, a layer of epithelial cells, acts as defender to protect against pathogens. Tight junctions (TJs), such as occludin and zonula occludens $1(\mathrm{ZO}-1)$, form the structurally critical foundation and increase intestinal mucosal barrier. Increased intestinal permeability is considered an early event in IBS-D and related to a reduction in the expression of occludin and ZO-1. Some studies have shown that colonization of germ-free animal with gut microbiota from patients with IBS-D can induce impaired intestinal permeability [5]. Metabolomic profiles, derived from gut microbiota, were the source of energy of intestinal epithelium to maintain the construction of barrier; hence, the dysbiosis may lead to dysfunction of intestinal barrier due to malnutrition [6].

Traditional Chinese medicine (TCM), as complementary and alternative medicine strategies, has been widely used for a long time in Asian countries to treat chronic diarrhea. For example, Ganodermataceae fungi are used as TCM for treating stomach diseases in China, and the study on cultivation conditions of Ganoderma with high nutritional and medicinal values 
Table 1 The composition of CKF

\begin{tabular}{llc}
\hline Chinese name & Latin name & $\begin{array}{l}\text { Dry weight of herbal } \\
\text { materials in CKF (g) }\end{array}$ \\
\hline Bai Shao & Paeoniae radix albe & 15 \\
Fang Feng & Saposhnikoviae radix & 6 \\
Shu Dihuang & Rehmanniae radix praeparata & 5 \\
Tu Sizi & Cuscutae semen & 5 \\
Jin Qiaomai & Fagopyri dibotryis rhizoma & 8 \\
Huang Lian & Coptidis rhizoma & 3 \\
Chan Tui & Cicadae periostracum & 3 \\
\hline Total & & 45 \\
\hline
\end{tabular}

is of economic benefits [7]. A multi-herb formula, CFK, is another TCM that has been used to treat chronic diarrhea for a long time. CKF, which is derived from an ancient traditional Chinese formula, is composed of 7 herbs, including Baishao (Paeoniae Alba Radix, the root of Paeonia lactiflora Pall.), Fang Feng (Total Fagopyri Dibotryis Rhizoma, the rhizome of Fagopyrum dibotrys (D.Don) Hara), Shu Dihuang (Saposhnikoviae Radix, the root of Saposhnikovia divaricate (Turcz.) Schischk.), Tu Sizi (Cuscutae Semen, the seed of Cuscuta chinensis Lam.), Jin Qiaomai (Rehmanniae Radix, the root of Rehmannia glutinosa Libosch.), Huang Lian (Coptidis Rhizoma, the rhizome of Coptis chinensis Franch.), and Chan Tui (Periostracum Cicadae, the exuviae of Cryptotympana pustulata Fabricius.). We have proved that CKF could introduce the remission of IBS-D in clinical trial. In addition, our experimental studies have shown that CKF ameliorates syndrome of IBS-D rats, which may relate to attenuate visceral hypersensitivity [8]. Using high-performance liquid chromatography (HPLC), we have determined active constituents in CKF, which include berberine, paeoniflorin, acteoside, flavonoids, and chromones [8].

Based on the aforementioned results, we hypothesize that CKF has a therapeutic effect on IBS-D by regulating gut microbiota. In the present study, we investigated the therapeutic effect of CKF by AWR, the fecal pellets, and the expression of ZO-1 in the colon. Moreover, the changes of the gut microbiota structure in IBS-D model rats after CKF treatment using 16S rRNA method were also observed.

\section{MATERIALS AND METHODS}

\section{Composition and preparation of $\mathrm{CKF}$}

All the seven raw powder medicinal herbs of CKF were purchased from Jiangsu Province Integration of Chinese and Western medicine (Nanjing, China), and their compositions in the CKF are shown in Table 1 . The raw powder herbs were soaked together in 10 volumes $(\mathrm{w} / \mathrm{v})$ of distilled water. The solution was heated to boil and maintained at $100^{\circ} \mathrm{C}$ for $1.5 \mathrm{~h}$. The decoction was filtered through a multi-layer gauze, and the residue was diluted with 8 volumes $(\mathrm{w} / \mathrm{v})$ distilled water for a second extraction. The filtrates obtained from the two extractions were combined for further uses.

\section{Animal}

All animal experiments were approved by the Experimental Animal Ethics Committee, Nanjing University of Chinese Medicine (Nanjing, Jiangsu, China). Male Sprague-Dawley rats (4 weeks old, weighed $140 \pm 10$ g) were purchased from Vital River Laboratories Animal Technology Co, Ltd (Beijing, China). Upon arrival, all rats were kept under an SPF environment at the animal center of Jiangsu Institute of Traditional Chinese Medicine and randomly transferred to plastic cage in an air-conditional room (temperature $23 \pm 3{ }^{\circ} \mathrm{C}$, humidity $55 \pm 5 \%$ ) under a 12 -h light/dark cycle. Water and standard chow were allowed ad libitum. All rats were kept for one week before being used in any experiment.

\section{Induction of IBS-D and treatment}

In this study, IBS-D model was established according to previously reported protocol with little modification [6] of psychosocial stress (restraint) combined with the peripheral stimulation stress (senna leaf gavage). In brief, the model group and the CKF group rats were gavaged with senna decoction $(3 \mathrm{~g} / \mathrm{kg}$ ) plus restraint stress for $2 \mathrm{~h}$ ( $1 \mathrm{~h}$ after the gavage) for 2 weeks. When the model was established, abdominal withdrawal reflex (AWR) scores were used to quantify the corio rectal distention (CRD), and the number of fecal pellets was used to evaluate the severity of diarrhea in each group. The control and the model groups were treated orally with distilled water. In the CKF group, the rats were given $2 \mathrm{ml} \mathrm{CKF}(18.8 \mathrm{~g} / \mathrm{kg}$ rat weight) per day via oral gavage. At the end of experiment (day 14), all rats were killed by cervical dislocation after the abdominal withdrawal reflex had been evaluated.

\section{Abdominal withdrawal reflex}

After 14 days of the treatment, the abdominal withdrawal reflex (AWR) scores were evaluated. The double-chambered pediatric end-balloon catheter (diameter $2.7 \mathrm{~mm}$ ) was coated with paraffin oil and connected to the syringe. The rats were anesthetized with isoflurane, and the balloon was inserted in the distal colon with the distal tip $1 \mathrm{~cm}$ from the anal verge. Then, $2 \mathrm{ml}$ of ambient temperature water were slowly injected into the balloon to observe the retreat of the abdominal wall and to induce AWR. The pressure of the balloon was increased in stepwise: 20,40,60, and $80 \mathrm{~mm} \mathrm{Hg}$ with a $30 \mathrm{~s}$ duration and repeated three times with 5-min intervals.

The AWR scores representing the behavioral responses were observed and evaluated. Briefly, rats were scored as follows: $0=$ the rats were emotionally stable and free to move; $1=$ the rats were emotionally 
unstable and occasionally twist their heads; $2=$ the abdominal muscles contracted slightly, but the abdomen did not lift off the ground; $3=$ the abdominal back muscles contracted strongly, and the abdomen was lifted off the ground; and $4=$ the abdominal back muscles contracted strongly, the back was arched, and the abdomen, the pelvis and the perineum were lifted off the ground.

\section{Immunohistochemistry}

After the rats were killed, the colon sections were rinsed with ice-cold PBS; excess PBS solution was removed by blotting; and the rinsed sample was immediately fixed in $10 \%$ buffered formalin, dehydrated, and paraffin embedded. Colon tissue sections (5 $\mu \mathrm{m}$ thick) were stained using standard immunohistochemical procedures. The tissue sections were incubated with a primary antibody ZO-1 (sc-33725, Santa Cruz) for $2 \mathrm{~h}$ at room temperature, followed by $30 \mathrm{~min}$ incubation with a pre-diluted horseradish peroxidaseconjugated secondary antibody (1:400). For negative controls, $1 \%$ non-immune serum in PBS replaced the primary antibodies. The immunohistochemistry staining of ZO-1 was scored by measuring the integrated optical density of at least three visions of each slice using Image pro-plus 6.0 software. ZO-1 antibody was purchased from Santa Cruz Biotechnology (sc-33725, Texas, USA).

\section{Microbial DNA extraction and PCR amplification}

After CKF treatment, feces of each rat were simultaneously obtained under sterile condition. Total genomic DNA was extracted from every stool sample using DNA Extraction Kit following the manufacturer's instructions. Quality and quantity of DNA was verified with NanoDrop and agarose gel. Extracted DNA was diluted to a concentration of $1 \mathrm{ng} / \mu \mathrm{l}$ and stored at $-20^{\circ} \mathrm{C}$ until further processing. The diluted DNA was used as template for PCR amplification of bacterial 16S rRNA genes with the barcoded primers and Takara Ex Taq (Takara Biotechnology Co., Ltd, Dalian, China). For bacterial diversity analysis, V3-V4 variable regions of 16S rRNA genes was amplified with universal primers 343F (5'-TACGGRAGGCAGCAG-3') and 798R ( $5^{\prime}$-AGGGTATCTAATCCT-3'). Amplicon quality was visualized using gel electrophoresis, purified with AMPure XP beads (Agencourt), and amplified for another round of PCR. After the purification with the AMPure XP beads, the final amplicon was quantified using Qubit dsDNA assay kit. Equal amounts of purified amplicon were pooled for subsequent sequencing analysis. Raw sequencing data were in FASTQ format. Paired-end reads were then preprocessed using Trimmomatic software to detect and cut off ambiguous bases $(\mathrm{N})$. Low quality sequences with average quality score below 20 were also cut off using sliding window trimming approach. After trimming, paired-end reads were assembled using FLASH software. Parameters of assembly were: $10 \mathrm{bp}$ of minimal overlapping, $200 \mathrm{bp}$ of maximum overlapping, and $20 \%$ of maximum mismatched rate. Denoising of sequences was further performed as follows: reads with ambiguous, homologous sequences or below 200bp were abandoned; reads with $75 \%$ of bases above Q20 were retained; and reads with chimera were detected and removed. These two steps were achieved using QIIME software (version 1.8.0).

\section{Statistical analysis}

Data are expressed as Mean \pm S.E.M. $p$-values calculated using one-way or two-way analysis of variance (ANOVA) for multiple comparisons or two-tailed Student's $t$-test for paired comparisons.

\section{RESULTS}

\section{CKF ameliorated symptoms of IBS-D rats}

Body weight change, number of fecal pellets, and AWR scores were observed to assess the induction of IBS-D in rats. After 14 days, the weight of IBS-D rats and the number of fecal pellets were less than that of the normal rats, and the AWR scores were increased in the IBS-D rats when compared with those in the normal (without IBS-D) group. The results, therefore, strongly supported the successful establishment of IBS-D rat model. When the IBS-D rats were treated without or with CKF for 14 days (Fig. 1A), the body weight changes in the treated groups were also significantly lower than that in the control group, and there was no difference of body weight changes in IBS-D rats treated with or without CKF (Fig. 1B). There was no difference in AWR scores among the groups for the $20 \mathrm{~mm} \mathrm{Hg}$ distention pressure, but the AWR scores were remarkably increased when the distention pressures were 40,60, and $80 \mathrm{~mm} \mathrm{Hg}$ in the model group. However, the AWR scores of the CKF group were significantly reduced at the 40,60 , and $80 \mathrm{~mm} \mathrm{Hg}$ distention pressure (Fig. 1C). Moreover, the number of fecal pellets of IBS-D rats significantly increased compared with that of the normal rats, while rats treated with CKF could produce fewer fecal pellets than the model group (Fig. 1D). All of these indicated that CKF could significantly ameliorate symptoms of IBS-D rats.

\section{CKF promoted the expression of ZO-1 in IBS-D rats}

Since ZO- 1 is the most important transmembrane proteins that impact the permeability of tight junctions in colonic tissues, we detected their expression by Immunohistochemical staining. As shown in our results, the content of ZO-1 was downregulated in the model group compared with that in the control (Ctrl) group, suggesting an impaired intestinal mucosal barrier function in the IBS-D rats. After the CKF treatment, the expression of ZO-1 was remarkably increased. The 
A

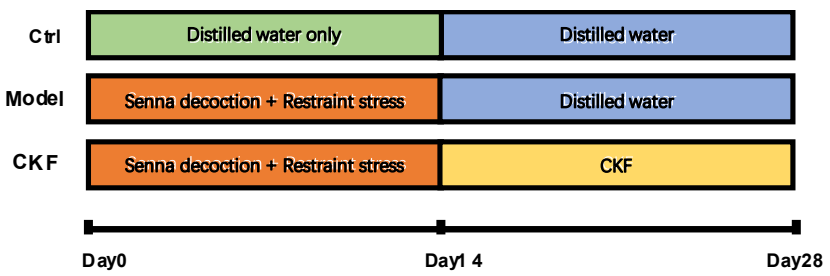

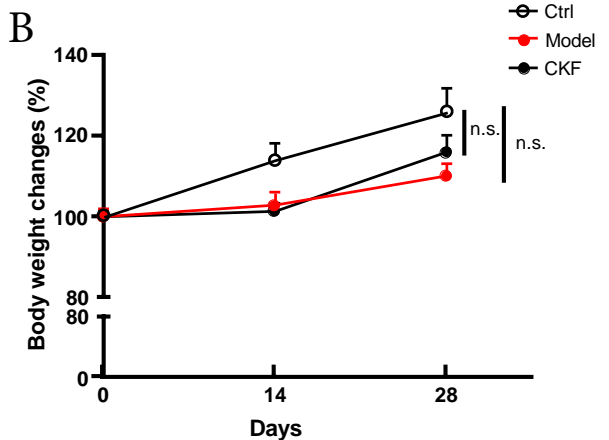

$\mathrm{D}$

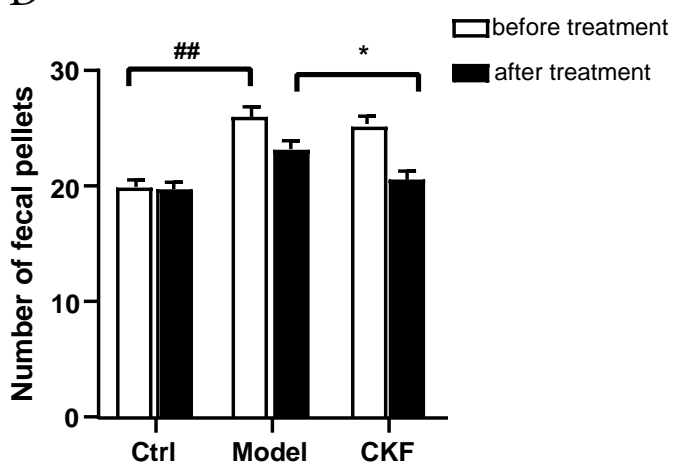

Fig. 1 CKF ameliorates symptoms in IBS-D rats. (A) Experimental design; (B) percentage of basal body weight changes during experimental duration; (C) AWR score measured on d28; (D) number of fecal pellets counted on d28. Results are mean \pm S.E.M. of six to eight rats in each group. $p$-values were calculated using one-way or two-way analysis of variance (ANOVA) for multiple comparisons or two-tailed Student's $t$-test for paired comparisons. ${ }^{\#} p<0.05$, ${ }^{\# \#} p<0.01$ versus normal; $* p<0.05$ versus model group.

A

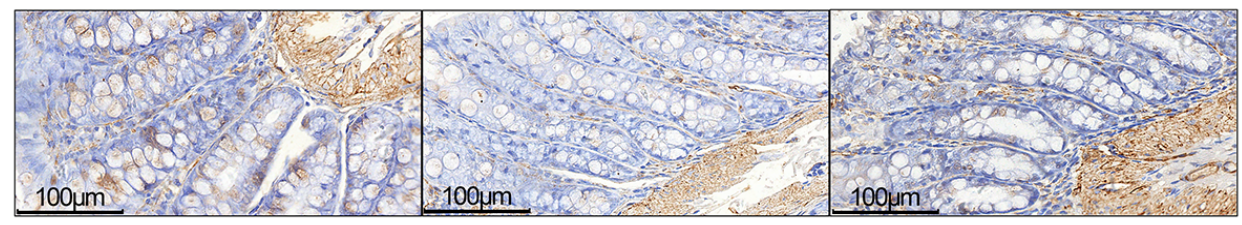

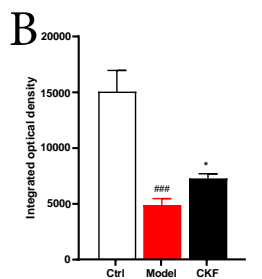

Fig. 2 CKF upregulated the expression of ZO-1 in IBS-D rats. (A) Representative images of immunohistochemistry staining for ZO-1 (brown) of colon tissue from different groups, bar $=100 \mu \mathrm{m}$; (B) quantitative analysis of ZO-1 in different groups. Results are mean \pm S.E.M. of six rats in each group. $p$-values were calculated using one-way or two-way analysis of variance (ANOVA) for multiple comparisons or two-tailed Student's $t$-test for paired comparisons. ${ }^{\# \# \# ~} p<0.001$ versus the normal group; * $p<0.05$ versus the model group.

above result strongly suggested that CKF could enhance intestinal mucosal barrier function in IBS-D rats (Fig. 2A,B).

\section{CKF reversed gut dysbiosis of the IBS-D rats}

Because of the vital role of gut microbiota in IBS, we then analyzed the structure of the gut microbiota of the different groups. Firstly, we noted that $\alpha$-diversity, the representative parameter of richness and diversity of flora as calculated by the Chao's index, was not significantly different between the Ctrl group and the Model group. However, the index was significantly upregulated after CKF treatment (Fig. 3A). Moreover, we calculated the Shannon's index and found that it was decreased in the IBS-D group when compared with the normal rats. Similar to the Chao's index, the Shannon's index is higher in the CKF group than in the other groups. As a result, hence, it means that the CKF could restore the diversity of gut microbiota (Fig. 3B). Principal coordinate analysis (PCoA) 
A
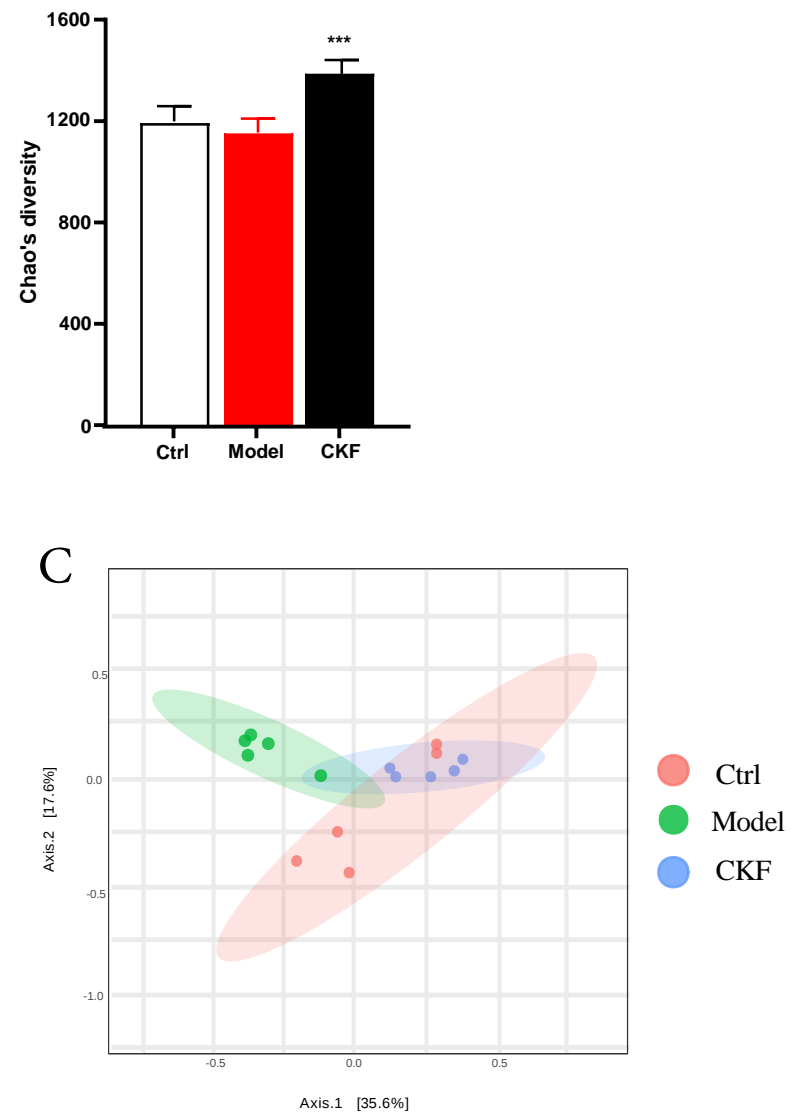

B

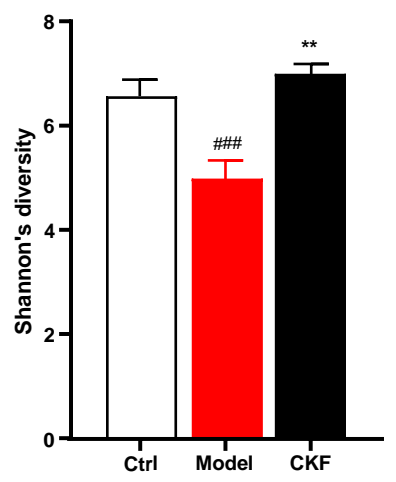

$\mathrm{D}$

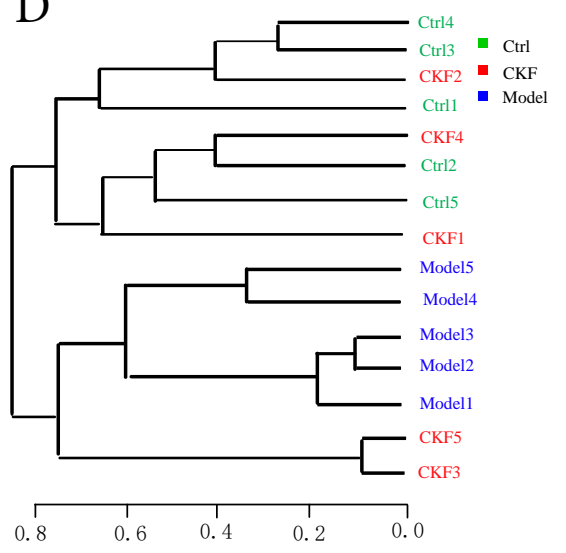

Fig. 3 CKF reversed gut dysbiosis of the IBS-D rats. (A) Gut microbial Chao's diversity analysis; (B) gut microbial Shannon's diversity analysis; (C) PCoA plot of fecal microbiota of each group; (D) multiple sample similarity tree. ${ }^{\# \# \# ~} p<0.001$ versus the normal group; $* * p<0.01,{ }^{* * *} p<0.001$ versus the model group.

revealed a distinct shift of composition gut microbiota in the IBS-D rats treated with CKF compared with the IBS-D rats (Fig. 3C). The system clustering tree showed that differences existed in the three groups, and the level of the CKF group was close to that of the Ctrl group (Fig. 3D).

\section{CKF regulated certain microbiota}

To understand the impact of CKF on gut microbiota, we then analyzed different taxonomic levels of gut microbiota in details. As shown in our results, at phylum level (Fig. 4A), nine kinds of gut microbiota were detected, and the most abundant phyla were Firmicutes and Bacteroidetes. In the IBS-D rats, the relative abundance of Firmicutes was decreased, while the abundance of Bacteroidetes was increased. However, after being treated with CKF, this phenomenon was reversed (Fig. 4B,C). At the family level (Fig. 4D), the relative abundances of Lachnospiraceae, Ruminococcaceae, and Lactobacillaceae were lower in the Model group, compared with the normal rats, but after the
CKF treatment, the relative abundances of the three kinds of microbiota were increased (Fig. 4E-G). Moreover, the relative abundance of Prevotellaceae was higher in the IBS-D rats, and the CKF reversed this phenomenon (Fig. 4H). At the genus level, the relative abundances of Allobaculum, Lactobacillus, Lachnospiraceae_NK4A136, and Lactobacillus, which are the short chain fatty acids (SCFAs) producers, were lower in the IBS-D rats compared with the normal rats, and the CKF treatment could increase these microbiota's levels in the IBS-D rats (Fig. 4I-L). Taken together, these results demonstrated that CKF could increase the numbers of bacteria in the IBS-D rats.

\section{DISCUSSION}

In this study, we established an IBS-D model rats through psychotic stress combined with peripheral stimulation stress (sennae gavage) for 2 weeks; and some of them were treated with CKF, while other were given the distilled water. As shown in our results, although there was no difference in body weight 

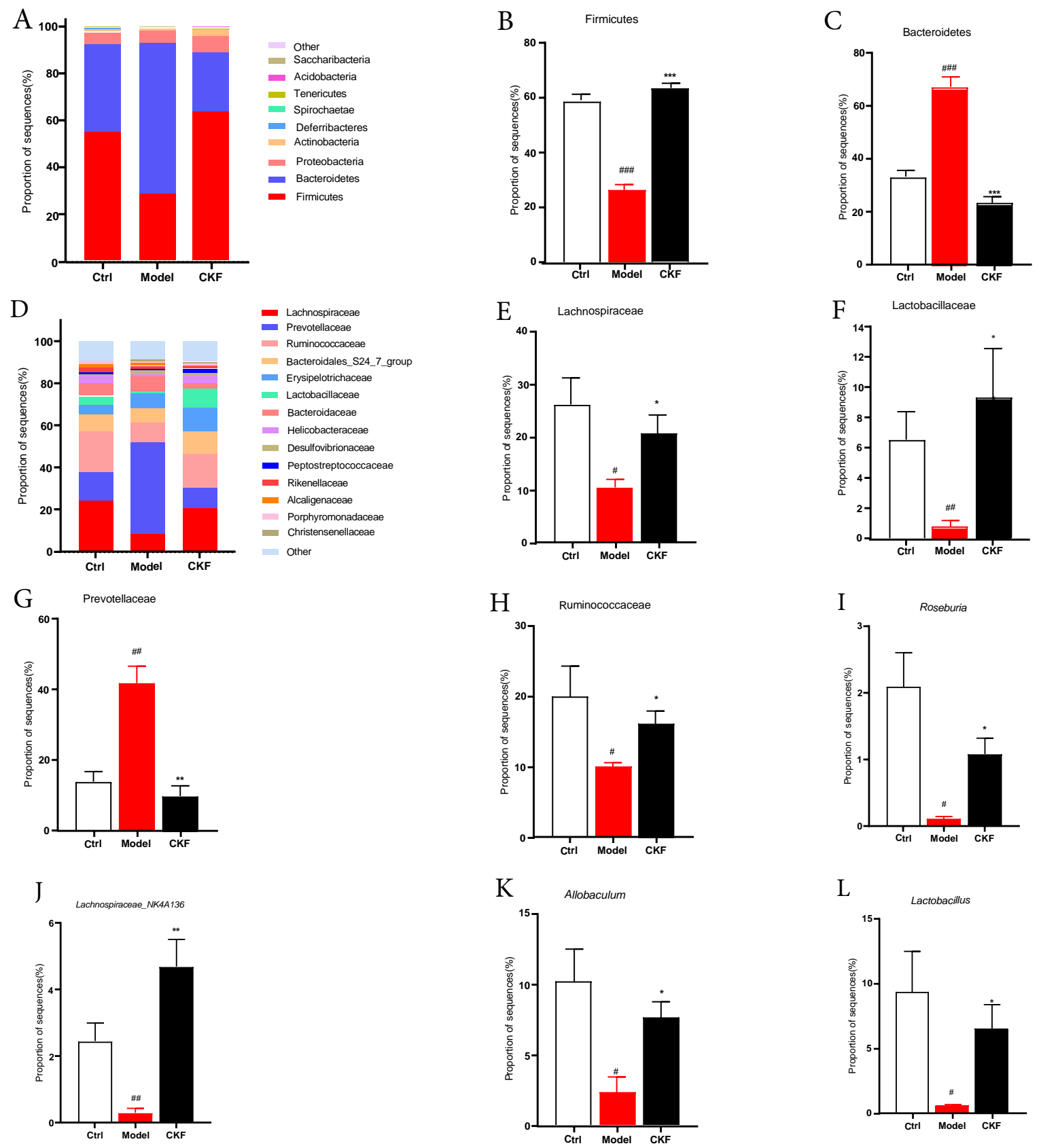

Fig. 4 CKF regulated a number of microbiota. (A) Stacked bar plot of the phylogenetic composition of common bacterial taxa at the phylum level; (B,C) relative abundance of Firmicutes (B) and Bacteroides (C) $(n=5)$; (D) stacked bar plot of the phylogenetic composition of common bacterial taxa at the family level; (E-H) relative abundances of Lachnospiraceae (E), Lactobacillaceae (F), Ruminoccoccaceae (G), and Prevotellaceae (H) $(n=5)$; (I-L) relative abundance of Roseburia (I), Lachnospiraceae_NK4A136 (J), Allobaculum (K), and Lactobacillus (L) at genus level $(n=5)$. Results are mean \pm S.D. of five rats in each group. $p$-values were calculated using one-way or two-way analysis of variance (ANOVA) for multiple comparisons or two-tailed Student's $t$-test for paired comparisons. ${ }^{\#} p<0.05$, ${ }^{\# \#} p<0.01$, ${ }^{\# \#} p<0.001$ versus the normal group; * $p<0.05$, ** $p<0.01, * * * p<0.001$ versus the model groups. 
changed between the CKF group and the model group, the number of fecal pellets in the CKF group was increased, indicating that the CKF could relieve diarrhea in these rats.

The intestinal barrier, containing the physical barrier, the secretory barrier and the immunological barrier, maintains the homeostasis by separating the internal milieu from the external environment [6]. The single layer of epithelial cells along the intestinal tract and the TJs formed by the ability of neighboring epithelial cells are critical to formation and maintenance of the intestinal barrier, controlling the movement of water and other small molecules from the intestinal lumen into the mucosa. TJs (consisting of a heterogeneous group of transmembrane proteins such as ZO-1, occludins, and claudins) play a major role in intestinal health and a decrease in expression and activity of $\mathrm{TJ}$ proteins, probably a bacterial-mediated mechanism and a pathogenic factor for IBS-D.

According to recent studies, dysregulation of gut microbiota associated with IBS-D. Compared with healthy controls, IBS patients have shown the lower abundance in Lactobacillus, Bifidobacterium, and Faecalibacterium prausnitzii $[9,10]$. Furthermore, disturbed microbiota structure is linked to co-morbidities of IBS, such as anxiety, depression, and migraine $[11,12]$. On clinical trials, Lactobacillus probiotics $[13,14]$ and multispecies probiotics [15], which contains the SCFAs-producing bacteria, have shown therapeutic effects on IBS-D patients. Rebalancing the gut microbiota could be a new treatment of IBS-D, which is similar to the theory of Chinese medicine, rebalancing the internal environment of human body to deal with IBS-D. In our present studies, we have found CKF could significantly rebalance the gut microbiota in IBS-D rats. Interestingly, our results demonstrated that the alpha diversity of the gut microbiota community was decreased in the IBS-D model group, which is in disagreement with previous studies $[16,17]$. This may be due to the different methods of building IBS-D Model. PCoA analysis revealed that the structures of gut microbiota in the CKF group were closed to that of the Ctrl group, and both are significantly distant with the IBS-D group rats, indicating that the beta diversity of gut microbiota of the IBS-D Model rats differed from that of the CKF-treated rats and the Ctrl rats. In phylum level, the abundance of Firmicutes was decreased in the IBS-D model rats, while CKF treatment could reverse this phenomenon. Besides, the CKF-treated rats could downregulate the abundance of Bacteroidetes, which was domain in gut microbiota of the IBS-D rat. In another word, the abundances of Lactobacillus, Allobaculum, Roseburia and Lachnospiraceae_NK4A136 were increased after the CKF treatment.

In conclusion, our findings validated the effects of CKF on IBS-D, and protecting the intestinal function and rebalancing the gut microbiota may contribute to release of this disease.

Acknowledgements: This work was supported by Major Science and Technology Project of Jiangsu Province Administration of Traditional Chinese Medicine (ZD201708); the Jiangsu Provincial "six talent peaks" High talents the fourteenth batch (WSN-040); the Natural Science Foundation of Jiangsu Province (BK20161608); Jiangsu Health Vocational College University level scientific research projects(JKC201958).

\section{REFERENCES}

1. Lovell RM, Ford AC (2012) Global prevalence of and risk factors for irritable bowel syndrome: a meta-analysis. Clin Gastroenterol Hepatol 10, 712-721.

2. Enck P, Aziz Q, Barbara G, Farmer AD, Fukudo S, Mayer EA, Niesler B, Quigley EM (2016) Irritable bowel syndrome. Nat Rev Dis Primers 2, ID 16014.

3. Ford AC, Sperber AD, Corsetti M, Camilleri M (2020) Irritable bowel syndrome. Lancet 396, 1675-1688.

4. Ford AC, Lacy BE, Talley NJ (2017) Irritable bowel syndrome. $N$ Engl J Med 376, 2566-2578.

5. Zhu HM, Li L, Li SY, Yan Q, Li F (2019) Effect of water extract from Berberis heteropoda Schrenk roots on diarrhea-predominant irritable bowel syndrome by adjusting intestinal flora. J Ethnopharmacol 237, 182-191.

6. Turner JR (2009) Intestinal mucosal barrier function in health and disease. Nat Rev Immunol 9, 799-809.

7. Yu C, Xi L, Zhao Y, Feng A, Zha L, Yang H, Chen M, Zhang Y, et al (2020) Artificial cultivation and nutritional evaluation of proteins from a wild Ganoderma gibbosum strain. ScienceAsia 46, 548-556.

8. Mao Q, Shi L, Wang ZG, Luo YH, Wang YY, Li X, Lu M, Ju JM, et al (2017) Chemical profiles and pharmacological activities of Chang-Kang-Fang, a multi-herb Chinese medicinal formula, for treating irritable bowel syndrome. J Ethnopharmacol 201, 123-135.

9. Vila VA, Imhann F, Collij V, Jankipersadsing SA, Gurry T, Mujagic Z, Kurilshikov A, Bonder MJ, et al (2018) Gut microbiota composition and functional changes in inflammatory bowel disease and irritable bowel syndrome. Sci Transl Med 10, ID eaap8914.

10. Liu HN, Wu H, Chen YZ, Chen YJ, Shen XZ, Liu TT (2017) Altered molecular signature of intestinal microbiota in irritable bowel syndrome patients compared with healthy controls: A systematic review and metaanalysis. Dig Liver Dis 49, 331-337.

11. Arzani M, Jahromi SR, Ghorbani Z, Vahabizad F, Martelletti P, Ghaemi A, Sacco S, TOgha M, et al (2020) Gutbrain axis and migraine headache: a comprehensive review. J Headache Pain 21, ID 15.

12. Kennedy PJ, Cryan JF, Dinan TG, Clarke G (2014) Irritable bowel syndrome: a microbiome-gut-brain axis disorder?. World J Gastroenterol 20, 14105-14125.

13. Nobaek S, Johansson ML, Molin G, Ahrne S, Jeppsson B (2000) Alteration of intestinal microflora is associated with reduction in abdominal bloating and pain in patients with irritable bowel syndrome. Am J Gastroenterol 95, 1231-1238.

14. Martoni CJ, Srivastava S, Leyer GJ (2020) Lactobacillus acidophilus DDS-1 and Bifidobacterium lactis UABla-12 improve abdominal pain severity and symptomology in 
irritable bowel syndrome: Randomized controlled trial. Nutrients 12, ID 363.

15. Labus JS, Sohn W, Lee OY, Lee SP, Lee KN, Jun DW, Yoon BC, Choi HS, et al (2014) Effect of multispecies probiotics on irritable bowel syndrome: a randomized, double-blind, placebo-controlled trial. $J$ Gastroenterol Hepatol 29, 52-59.

16. Labus JS, Hollister EB, Jacobs J, Kirbach K, Oezguen N,
Gupta A, Acosta J, Luna RA, et al (2017) Differences in gut microbial composition correlate with regional brain volumes in irritable bowel syndrome. Microbiome 5, ID 49.

17. Li J, Cui H, Cai Y, Lin J, Song X, Zhou Z, Xiong W, Zhou $\mathrm{H}$, et al (2018) Tong-Xie-Yao-Fang regulates 5-HT level in diarrhea predominant irritable bowel syndrome through gut microbiota modulation. Front Pharmacol 9, ID 1110. 